\title{
Modulation of the Serum Cytokine Expression Pattern in Hymenoptera Allergic Patients Treated with Specific Venom Immunotherapy
}

\author{
Catalina Sanz ${ }^{1}$, María Isidoro-García ${ }^{*}, 2$, Ignacio Dávila ${ }^{3}$, Marién Pascual $^{3}$, Belén García Berrocal ${ }^{2}$ \\ and Félix Lorente ${ }^{4}$
}

${ }^{I}$ Department of Microbiology and Genetics, University of Salamanca, Spain

${ }^{2}$ Department of Clinical Biochemistry, University Hospital of Salamanca, Spain

${ }^{3}$ Department of Allergy, University Hospital of Salamanca, Spain

${ }^{4}$ Department of Pediatrics, University of Salamanca, Spain

\begin{abstract}
Venom immunotherapy (VIT) is an adequate model to explore the immune mechanisms underlying this type of treatment. We have investigated the use of protein arrays to detect variations in the levels of cytokines in patients receiving VIT. In the present study we selected 11 non-atopic patients with systemic reactions after Hymenoptera sting that received VIT during at least three years. In order to evaluate the success of VIT all of them should have tolerated a sting field after VIT. Serum samples were obtained before initiating VIT and after at least three years of successful VIT. We analyzed 42 serum proteins corresponding to a Th1/Th2 panel using protein array methodology. We observed a significant increase of Interleukin 10, Myeloid Macrophage Colony Stimulation Factor, Macrophage Derived Chemokine, Interleukin 1- $\alpha$, Vascular Endothelial Growing Factor and Stem Cell Factor serum levels after successful VIT. We discuss the usefulness and normalization of this array method to analyze cytokines and other serum proteins. Monitoring these serum cytokines could help to predict the response and to elucidate the mechanisms underlying immunotherapy.
\end{abstract}

Keywords: Array, cytokine, hymenoptera, immunotherapy, allergy, interleukin.

\section{INTRODUCTION}

Hymenoptera hypersensitivity has not only served as a clinical model of anaphylaxis but also to explore mechanisms underlying specific immunotherapy. Several classic immunological changes that appear during immunotherapy have been clearly demonstrated in patients undergoing venom immunotherapy (VIT), for example the increase in venom specific Immunoglobulin $\mathrm{G}(\mathrm{IgG})$ levels or the changes in the affinity of IgG and Immunoglobuline $\mathrm{E}$ (IgE) antibodies [1]. In addition, changes in the cytokine pattern [2] and the induction of T-cell unresponsiveness during VIT [3] have been described. More recent studies have reported the induction of Interleukin - 10 (IL-10) and Interferon alpha (IFN $\alpha$ ) in patients treated with VIT [4] and the appearance of regulatory $\mathrm{T}$ cells during the course of VIT [5].

The array methodology consists in an ordered immobilization of multiple biomolecules in a tiny solid surface, allowing their identification and quantification, and therefore the acquisition of a huge quantity of information in a single experiment. The first arrays were designed to obtain information about the expression of several genes through the quantification of the corresponding RNA transcripts [68]. Rapidly, several modifications were introduced and

*Address correspondence to this author at the Department of Clinical Biochemistry, University Hospital of Salamanca, Paseo de San Vicente 58, 37007 Salamanca, Spain; Tel: 34923 291373; Fax: 34923 294593;

E-mail: misidoro@usal.es protein arrays appeared [9]. This methodology has allowed to study multiple proteins from different biological samples, to investigate complete proteomes and to design diagnostic applications [10-13].

The use of protein arrays in the field of asthma and allergy is an attractive challenge since these entities are multi-factorial inflammatory diseases in which the release of hundreds of proteins does occur. It has been reported an urgent need to identify and validate predictive biomarkers that can be used to monitor allergen immunotherapy [14]. In this study, we decided to investigate the use of protein arrays to detect variations in the serum level of a specific panel of Th1/Th2 (type 1 helper $T$ cells / type 2 helper $T$ cells) cytokines during the course of successful immunotherapy. This could help to predict the response and to further elucidate the mechanisms underlying immunotherapy.

\section{MATERIALS AND METHODS}

\section{Patients}

Patients that strictly fulfilled the following requisites were included in the study: (i) a previous diagnosis of systemic reaction to hymenoptera sting; (ii) the time course of VIT was at least three years of specific VIT to the hymenopter that produced the reaction; (iii) after at least three years of VIT a field sting with the involved hymenopter should have produced no systemic reaction; (iv) negative skin prick test to a battery of aeroallergens in order to avoid atopy as a possible confounding factor. All patients were 18 years older and signed an informed written consent. The study was performed after the approval and following 
the recommendations of the Ethics Committee of the University Hospital of Salamanca and The Code of Ethics of the World Medical Association (Declaration of Helsinki).

All patients had suffered a systemic reaction after a Hymenoptera sting. The diagnosis and treatment of hymenoptera sensitivity was performed following the European Academy of Allergology and Clinical Immunology (EAACI) Interest Group on Insect Venom Hypersensitivity recommendations $[15,16]$. Serum samples before and after VIT were obtained, aliquoted and frozen at $80^{\circ} \mathrm{C}$. Aliquots were not thawed more than twice.

Skin prick tests with a battery of common aeroallergens were performed to all patients in order to determine whether the patient was also atopic [17]. Total IgE and specific IgE levels against the venoms of Apis mellifera, Vespula sp and Polistes $s p$ were determined by enzyme immunoassays following manufacturer's recommendations (Phadia CAP system, Uppsala, Sweden).

\section{Array Assay}

Serum levels of the following proteins were simultaneously analyzed: Epithelial neutrophil-activating protein 78 (ENA-78), Granulocyte-colony stimulating factor (GCSF), Granulocyte-macrophage colony stimulating factor (GM-CSF), Growth related oncogene (GRO), Growth related oncogene-alpha (GRO $\alpha$ ), I-309 (I-309), Insulin-like growth factor-1 (IGF-1), Interleukin 1 alpha (IL-1 $\alpha$ ), Interleukin 1 beta (IL-1 $\beta$ ), Interleukin 2 (IL-2), Interleukin 3 (IL-3), Interleukin 4 (IL-4), Interleukin 5 (IL-5), Interleukin 6 (IL6), Interleukin 7 (IL-7), Interleukin 8 (IL-8), Interleukin 10 (IL-10), Interleukin 12 (IL-12p40p70), Interleukin 13 (IL13), Interleukin 15 (IL-15), Interferon alpha (INF $\alpha$ ), Monocyte chemoattractant protein 1 (MCP-1), Monocyte chemoattractant protein 2 (MCP2), Monocyte chemottractant protein 3 (MCP-3), Macrophage-colony stimulating factor (MCSF), Macrophage-derived chemokine (MDC), Monokine induced by gamma interferon (MIG), Macrophage inflammatory protein 1 delta (MIP-1 $\delta$ ), Regulated upon activation, normal T-cell expressed, and presumably secreted (RANTES), Stem cell factor (SCF), Stromal cell-derived factor (SDF-1), Thymus and activation-regulated chemokine (TARC), Tumor growth factor beta 1 (TGF $\beta 1$ ), Tumor necrosis factor-alpha (TNF $\alpha$ ), Tumor necrosis factor-beta (TNF $\beta$ ), Epidermal growth factor (EGF), Insulin-like growth factor-1 (IGF-I), Angiogenin, Oncostatin M, Trombopoyetin, Vascular endothelial growth factor (VEGF), Platelet-derived growth factor BB (PDGFBB) and Leptin. The RayBio Human Cytokine Antibody Array III was employed for the determinations, following manufacturer's recommendations (Raybiotech, Inc; www.raybiotech.com) (Fig. 1). Each membrane included six positive controls and six negative controls. In addition, Leptin was determined by EnzymeLinked Immunoabsorbent Assay (ELISA) technique (MEDIAGNOST, Reutlingen, Germany).

To avoid non-specific binding membranes were initially blocked with a blocking solution (10\% dilution of casein in Maleic acid), for 30 minutes at room temperature and they were overnight incubated with sera at $4^{\circ} \mathrm{C}$ with constant shaking. Membranes were then washed with washing buffer. Biotin-conjugated anti-cytokine primary antibody diluted in Blocking Buffer was added and membranes were incubated at room temperature for 2 hours. After that, membranes were washed and incubated at room temperature for 2 hours with a solution of streptavidin tagged to peroxidase, and washed again with wash buffer. Finally, a chemoluminiscent Detection Buffer was added. Membranes were incubated for 5 minutes at room temperature and exposed to a Kodak XOMAT AR film. Images were also processed in a chemoluminescence imaging device (Luminescent Image Analyzer LAS-1000plus, Fujifilm, Stamford, USA).

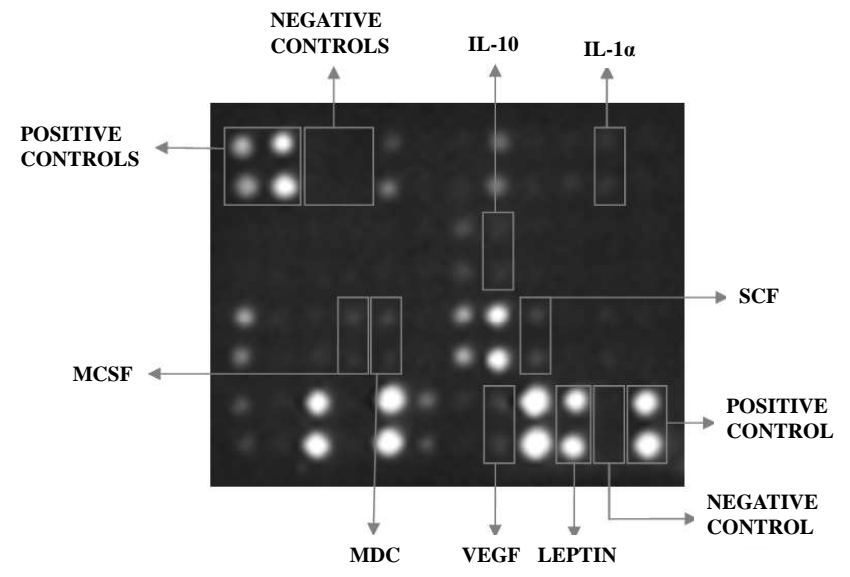

Fig. (1). RayBio Human Cytokine Antibody Array III. Example of one RayBio Human Cytokine Antibody Array III detection where it is indicated the position of the positive and negative controls, as well as the array localization of the cytokines for which a significant difference between the pre VIT and the post VIT determinations was detected.

For each patient, samples were collected before and after specific VIT. Samples were analyzed by duplicate in different membranes (interassay analysis) and each protein was analyzed twice in each membrane (intraassay analysis). A total of four determinations of each protein were performed for each condition.

In order to confirm the putative effects of time course over the IL-10 expression, serum levels of IL-10 were analyzed in a group of 23 untreated patients with the BioPlex ${ }^{\circledR}$ suspension array system (Bio-rad, Hercules, CA, USA) following the manufacture's recommendations. For each patient a sample was collected at an initial stage and another sample was collected six month later. Both samples were stored at $-80^{\circ} \mathrm{C}$ after being extracted and were analyzed simultaneously for IL-10 determination. For each patient and each time two determinations were performed (four determinations for each patient).

\section{Data Analysis}

The signals from the array analysis were processed with Image Reader LAS-1000 (Fujifilm, Stamford, USA) and SpotReader (Niles Scientific, San Francisco, USA) software. For each spot, an intensity value of the signal and a value that included the background signal were obtained. An intensity threshold was calculated with the mean plus two standard deviations of negative values. The spots that did not surpass the intensity threshold were excluded of the analysis after the normalization process.

The ratios from replicate antibody measurements within the same array were averaged. For normalization of the 
method, the averaged ratios were multiplied by a normalization factor $\mathrm{N}$ for each array. This factor was calculated by $\mathrm{N}=\left(\mathrm{S}_{\mathrm{L}} / \mu_{\mathrm{L}}\right) / \mathrm{R}_{\mathrm{L}}$ where $\mathrm{S}_{\mathrm{L}}$ is the ELISA-measured Leptin concentration of the serum sample on that array; $\mu_{\mathrm{L}}$ is the mean ELISA-measured Leptin concentration of all the samples; $\mathrm{R}_{\mathrm{L}}$ is the average ratio of the replicate antibody spots on the array [18]. The Mean Centering method was also applied for normalization. The averaged ratios were multiplied by a normalization factor $\mathrm{N}$ for each array that was calculated by $\mathrm{N}=1 / \mu$, where $\mu$ is the mean ratio of all antibody spots on the array [18].

Intra-assay precision and Inter-assay precision were also analyzed for this methodology. The coefficient of variation and analysis of correlation were used for this study. Signal differences between cytokine levels before and after immunotherapy were evaluated applying the Wilcoxon Signed Ranks test. SPSS 12 program was used for statistical analysis. A p-value of less than 0.05 was considered statistically significant. Statistical power of the study was also calculated for this sample size. 0.05 was considered as alfa error (http://www.dssresearch.com/toolkit/sscalc/size_a2.asp).

\section{RESULTS AND DISCUSSION}

In this study, we have analyzed 42 different proteins in 22 serum samples (a total of 924 determinations) corresponding to the 11 patients treated with VIT. Samples were analyzed in quadruplicate, duplicates for the intra-assay and duplicates for the inter-assay procedures, with a total amount of 4224 spots analyzed, positive and negative controls included (Fig. 1).

ELISA analysis of Leptin was independently performed as internal standard. The normalization of each array was performed by setting the internal standard to the standard's known values. The effects of different approaches in the normalization procedure were evaluated.

The repeatability between replicate data sets was analyzed by examining both the coefficients of variation (CV) and the correlations between the replicate experiments. The CV of each antibody between the quadruplicate measurements was calculated. The average CVs were compared between the intra and inter-assay. As expected, the average CVs were significantly higher $(\mathrm{p}<0.001)$ in the interassay $(0.27 \pm 0.24)$ than in the intra-assay $(0.06 \pm 0.06)$ analysis.

The average of the intra-assay correlations was significantly higher when we consider the signal (0.94 \pm $0.07)$ than when we consider jointly the signal and its background $(0.82 \pm 0.13), \mathrm{p}<0.0001$ (Table 1). After the normalization procedure the average of correlations increased significantly $(0.98 \pm 0.02)(p=0.01)$.

The average of the inter-assay correlations considering just the intensity of the signal $(0.90 \pm 0.05)$ was also higher than considering jointly the signal and its background (0.89 \pm 0.11 ), but these differences did not reach statistical signification (Table 2). We also observed a higher interassay correlation when the exclusion of the uncertainty points was performed after the normalization process. Correlations of the non-normalized data were compared with the correlations of the normalized data (Tables $\mathbf{1}$ and 2 ).
Correlations were also significantly improved after the normalization process.

Table 1. Repeatability of Data Across Arrays; Intra-Assay Correlations

\begin{tabular}{|c|c|c|}
\hline & $\begin{array}{l}\text { antra-Assay } \\
\text { Correlations }\end{array}$ & 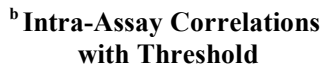 \\
\hline Signal intensity & $* 0.94 \pm 0.07$ & $* * 0.91 \pm 0.19$ \\
\hline Signal and Background & $0.82 \pm 0.13$ & $0.84 \pm 0.12$ \\
\hline Normalized data & $\dagger 0.98 \pm 0.02$ & $\dagger \dagger 0.98 \pm 0.02$ \\
\hline
\end{tabular}

Mean \pm standard deviation of correlations

${ }^{b}$ Mean \pm standard deviation of correlations when the threshold is applied.

*Wilcoxon $\mathrm{p}<0.0001$ for the comparison of the intra-assay correlation of the signal intensity with the intra-assay correlation of the signal with its background.

$* *$ Wilcoxon $p<0.0001$ for the comparison of the intra-assay correlation of the signal intensity with the intra-assay correlation of the signal with its background when the threshold is applied.

$\dagger$ Wilcoxon $\mathrm{p}=0.01$ for the comparison of the intra-assay correlation of the signal intensity with the Normalized data.

$\dagger+$ Wilcoxon $p=0.011$ for the comparison of the intra-assay correlation of the signal intensity with the Normalized data when the threshold is applied.

Table 2. Repeatability of Data Across Arrays; Inter-Assay Correlations

\begin{tabular}{|c|c|c|}
\hline & $\begin{array}{c}{ }^{\mathrm{a}} \text { Inter-Assay } \\
\text { Correlation }\end{array}$ & $\begin{array}{c}{ }^{\mathrm{b}} \text { Inter-Assay Correlation } \\
\text { with Threshold }\end{array}$ \\
\hline \hline Signal intensity & $0.90 \pm 0.05$ & $0.90 \pm 0.05$ \\
\hline Signal and Background & $0.89 \pm 0.11$ & $0.88 \pm 0.13$ \\
\hline Normalized data & $0.90 \pm 0.06$ & $0.90 \pm 0.06$ \\
\hline
\end{tabular}

${ }^{\mathrm{a}}$ Mean \pm standard deviation of correlations.

${ }^{b}$ Mean \pm standard deviation of correlations when the threshold is applied after normalization.

In our study, a very well defined population was selected. Patients were followed during five years and all of them received at least three years of specific VIT. In addition, all patients tolerated a sting field, indicating that VIT had been successful. Atopic patients were excluded to avoid possible confounding factors due to an immunological response to other allergens. In our opinion, this is an appropriate model to analyze the immunological changes that account during specific IT (Immunotherapy). Previous studies focused in specific cytokines have been reported. Thus, de Amici et al., [19] determined serum levels of IL-1 $\alpha$, IL-2, IL-6, and TNF $\alpha$ on peripheral blood samples of 11 allergic patients with respiratory symptoms (asthma and/or rhinitis) before and after 3, 6, and 9 months of specific immunotherapy. Recently, Jerzyńska et al., [20] investigated serum levels of IL-1 $\alpha$, IL-6, and TNF $\alpha$ in 32 children with allergic asthma before and after 3 and 12 months of VIT.

For this analysis, we employed an array system to study a specific Th1/Th2 panel. Firstly a methodological optimization of the system was performed by combining this method with the ELISA assay as standard. The use of a precise and reliable method for data treatment is essential for an adequate interpretation of the huge amount of information obtained from the assay. The experience on array technology accumulated during the last 15 years has revealed that normalization must be properly used to avoid errors and 
scientific misleading [21]. Recent studies have shown that array data were incorrectly analyzed many times [22].

Normalization is the way of correcting possible systematic experimental variation factors to avoid the impact of non-biological effects on the biological data. Hamelinck et al., [18] recently undertook a study to determine the optimal normalization of data from antibody microarray profiling of proteins in human serum specimens. They compared seven different normalization methods representing major classes of normalization for antibody microarray data by their effects on reproducibility, accuracy, and trends in the data set. Some of the methods performed well in one category but not very well in another, showing the value of using multiple criteria for the evaluation. Therefore, putting all of the information together, the ELISA normalization method seems to perform the best. In our study, an analysis of repeatability was performed. The best results were obtained, as expected, in the intra-assay analysis. The correlation analysis was significantly improved after the normalization process and the best results were obtained when the exclusion of the uncertainty points was done after the normalization process.

In this study, we detected a significant increase of IL-10, (Wilcoxon $\mathrm{p}=0.036$ ) (Table 3 and Fig. 2), IL-1 $\alpha$, MCSF, VEGF, MDC and SCF in the sera of patients that received
VIT. To discard the putative effects of time over the cytokine expression pattern in the serum samples, a control group 23 untreated patients was analyzed. The geometric mean of IL-10 levels corresponding to the 23 patients sample at the first stage was 3.12 detection units, whereas the same measure for the second stage was 1.67 detection units, although no statistical differences were detected. IL-10 is an anti-inflammatory cytokine that suppresses both Th1 and Th2 responses. It promotes B-cell activation and regulates immunoglobulin class switching. In addition, IL-10 inhibits the production of IL-1 $\alpha$, IL-6, IL-8, IL-12, and TNF $\alpha$ by mononuclear phagocytes and the production of IFN $\alpha$ and $\mathrm{TNF} \alpha$ by natural killer (NK) cells. It can also induce the appearance of regulatory $\mathrm{T}$ cells [23]. Thus, during allergen immunotherapy IL-10 contributes to the development of tolerance, regulates specific isotype formation and skews the specific response from an IgE-dominated to an IgG4dominated phenotype [24]. In this sense, up-regulation of IL10 has been demonstrated following venom immunotherapy [4]. In addition, Nasser et al., reported an increase of IL-10 levels in cutaneous biopsies of patients undergoing wasp venom immunotherapy. Furthermore, PBMC from beekeepers who are hyper-immune to bee stings produces increased IL-10 in response to stimulation with bee venom allergen [25]. In our model of successful VIT, the increase of

Table 3. Signification of Differences Between Pre and Post Immunotherapy for All Cytokines Analyzed Before and After Excluding Data that do Not Pass the Threshold

\begin{tabular}{|c|c|c|c|c|c|}
\hline Cytokine & Wilcoxon-p Before Threshold & Wilcoxon-p After Threshold & Cytokine & Wilcoxon-p Before Threshold & Wilcoxon-p After Threshold \\
\hline ENA-78 & 0.131 & 0.176 & МCP-2 & 0.026 & 0.109 \\
\hline GM-CSF & 0.050 & 0.068 & MCSF** $^{* *}$ & 0.041 & 0.028 \\
\hline GRO & 0.041 & 0.093 & MDC** & $\mathbf{0 . 0 3 3}$ & 0.012 \\
\hline I-309 & 0.033 & 0.068 & MIP-1 & 0.374 & 0.515 \\
\hline IL-1 $\alpha * *$ & 0.033 & 0.043 & RANTES & 0.929 & 0.878 \\
\hline IL-1 $\beta$ & 0.041 & - & SCF** & 0.021 & 0.018 \\
\hline IL-2 & 0.075 & - & SDF-1 & 0.026 & 0.285 \\
\hline IL-6 & 0.091 & 0.345 & TNF $\beta$ & 0.033 & 0.109 \\
\hline IL-7 & 0.131 & 0.144 & EGF & 0.328 & 0.445 \\
\hline IL-8 & 0.050 & 0.263 & IGF-1 & 0.033 & 0.068 \\
\hline IL10** & 0.026 & 0.036 & Ang & 0.859 & 0.929 \\
\hline IL-12 & 0.041 & 0.285 & OSM & 0.075 & 0.139 \\
\hline Il-13 & 0.026 & 0.285 & TPO & 0.041 & 0.068 \\
\hline IL-15 & 0.041 & 0.655 & VEGF** & 0.026 & 0.018 \\
\hline INF $\gamma$ & 0.062 & - & PDGF B & 1 & 1 \\
\hline
\end{tabular}

${ }^{* *}$ Cytokines with a Wilcoxon $\mathrm{p}$ value $<0.05$ between pre and post immunotherapy after applying threshold. 
serum IL-10 levels is in agreement with these data and could serve to monitor immunotherapy.

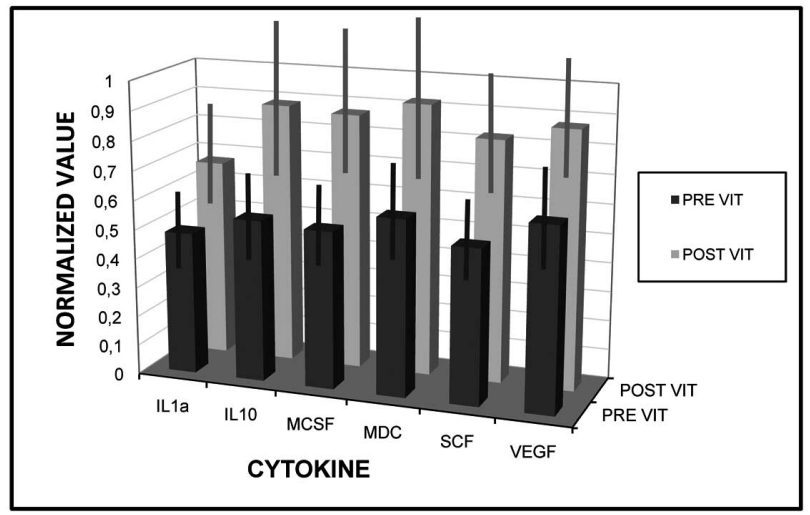

Fig. (2). Serum levels of array proteins differentially expressed pre and post VIT. Serum levels of Interleukin-1 $\alpha$ (IL-1 $\alpha$ ), Interleukin10 (IL10), Macrophage-colony Stimuating Factor (MCSF), Macrophage-derived Chemokine (MDC), Stem Cell Factor (SCF) and Vascular Endothelial Growth Factor (VEGF) analyzed pre and post Venom Immunotherapy (VIT).

We also noticed a significant increase of Interleukin $1 \alpha$ (IL-1 $\alpha$ levels in the sera of patients that received VIT (Wilcoxon $p=0.043$ ) (Table 3, Figs. 2, 3). To certain extend some previous reported data related to IL- $1 \alpha$ serum levels after VIT are contradictory. Thus De Amici et al., [19] found an increase in serum levels of IL- $1 \alpha$ after nine months of VIT, whereas Jerzyńska et al., [20] found no modification of serum IL- $1 \alpha$ levels after 12 months of VIT. IL- $1 \alpha$ is a potent inducer of IL-10 and also plays a role in early T-cell priming, which has been considered to be one key point of interest in the development of tolerance. Karjalainen et al., [26] showed that the $I L I A$ and $I L 10$ genes were substantially related to the development of cat exposure-induced tolerance. The associations of $I L I A$ and $I L 10$ illustrated in their study indicate that exposure-related tolerance can be a biological phenomenon with demonstrable genetic background. It further remains to be elucidated whether the $I L 1 A$ and IL10 genotypes mediate the responses in induced tolerance (i.e., in specific immunotherapy) [19].

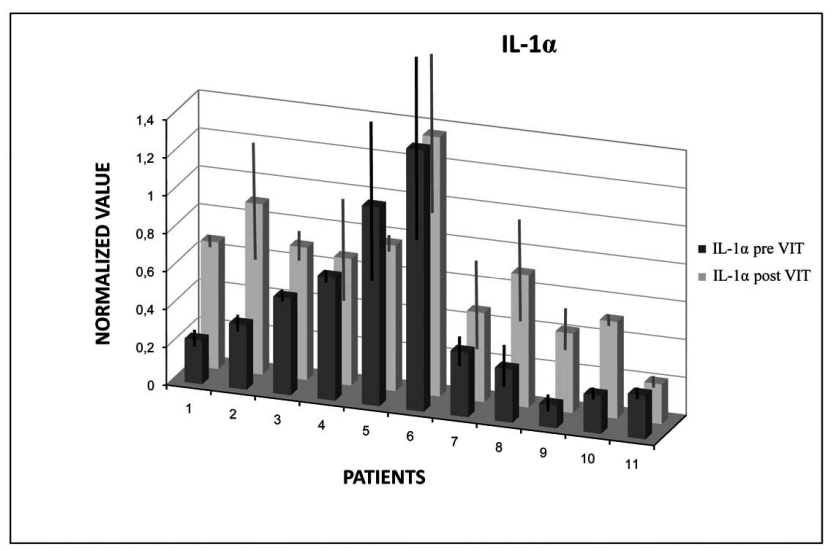

Fig. (3). IL-1 $\alpha$ serum levels pre and post VIT. Serum levels of Interleukin $1 \alpha(\mathrm{IL}-1 \alpha)$ analyzed in 11 patients pre and post Venom Immunotherapy (VIT).

In this study, an increase of Myeloid Macrophage Colony Stimulation Factor (MCSF) was also observed after immunotherapy (Wilcoxon $\mathrm{p}=0.028$ ) (Table $\mathbf{3}$ and Fig. 2).
Increased levels of M-CSF in response to IL- $1 \alpha$ have been detected in cell culture [27]. In addition, IL-1 $\alpha$ can increase the production of M-CSF by vascular cells [28]. It has been reported that M-CSF-conditioned dendritic cell (DC) precursors can participate in the modulation of inflammation and immune response by rapid release of IL-10 [29]. The authors described that M-CSF-conditioned human DC precursors responded to LPS, Mycobacteria bovis, and inflammatory cytokines by a rapid and robust production of IL-10. It has been proposed a synergistic enhancement of IL2-driven T-cell proliferation induced by CSF [30]. In addition, CSF also co-stimulated the production of interferon (IFN)-gamma by activated T-cells.

An increase of serum Vascular Endothelial Growing Factor (VEGF) levels was also detected in our patients (Wilcoxon $\mathrm{p}=0.018$ ) (Table 3 and Fig. 2). It has been reported that, in vivo, M-CSF increases VEGF mRNA expression in skeletal muscles. Although other authors described an increase in growth factors involved in angiogenesis after allergen exposure in non asthmatic patients with allergic rhinitis [31]. Studies of VEGF during VIT are sparse. Recently, Ciprandi et al., found the same "paradoxical" increase in VEGF levels after sublingual immunotherapy [32] which is in agreement with our findings.

Increased levels of Macrophage Derived Chemokine (MDC) were also detected (Wilcoxon $\mathrm{p}=0.012$ ) (Table 3 and Fig. 2). MDC is a potent chemoattractant for $\mathrm{Th} 2$ cells and has been implicated in Th2 type inflammation associated with the development of airway hyperresponsiveness [33, 34]. MDC is processed by the surface serin protease dipeptidylpeptidase IV/CD26 [35, 36]. This protease removes the N-terminal dipeptyde gly-pro. Subsequently, MDC (3- 69) is processed by removal of the tyr-gly dipeptide with generation of MDC (5-69). CD26-processed MDC (5-69) lost the capacity to interact with CCR4 and had little chemotactic activity on lymphocytes and dendritic cells. CD26 has been shown to be expressed preferentially and selectively on polarized type I T cells (compared with polarized type II Th2 cells) generating MDC (3- 69) and MDC (5-69), which do not attract type II T cells and do not interact with CCR4 [37]. In our array the MDC antibody could simultaneously detect the CD26-processed MDC. We have found no data about levels of MDC during VIT.

We have also detected a significant increase of soluble Stem Cell Factor (SCF) levels in patients after successful immunotherapy (Wilcoxon $\mathrm{p}=0.018$ ) (Table 3 and Fig. 2). Allergic diseases are characterized by a T-cell dependent increase of mast cells. SCF is the most important cytokine responsible for proliferation and mast cell growth. SCF is the ligand of c-Kit receptor and it is synthesized from two alternatively spliced mRNAs as transmembrane proteins. These proteins may be cleaved to produce soluble forms or cellassociated molecules [38]. It has been reported that the c-kit receptor is downregulated by the ligand. The ligand complexes are expected to be endocytosed leading to the down-modulation of the receptor molecules from the cell surface after ligand binding what may serve as a process to desensitize target cells [39]. In addition, it has been described that membrane-bound SCF prolongs c-Kit signaling whereas soluble SCF impedes signaling via c-KIT [40]. 
With this experimental choice we can detect putative variations in the serum levels of the cytokines in the patients treated with successful immunotherapy. Due to the restricting criteria of inclusion in this study, the sample size is limited; therefore the statistical power was particularly considered. The statistical power was more than $80 \%$ (alpha error of 0.05 ) for IL-1 $\alpha$ and SCF results, considering our population as reference. Indeed, the statistical power was under $80 \%$ for the rest of the cytokines that are mentioned. This is mainly due to the fact that the statistical power is greatly influenced by the sample size and in this type of studies is awarded the analysis of a large number of proteins in relatively small but well characterized samples. In our case, 11 patients were followed for a period of 5 years, immunotherapy was administered for at least 3 years and in all cases it was found that patients became tolerant to a sting after receiving the immunotherapy treatment. In addition to the strict clinical characterization, strict experimental rigor was maintained, carrying out more than four thousand determinations. For each patient and each situation the measurement was performed in quadruplicate and all data were subjected to strict normalization criteria. We detected significant differences for 24 cytokines, of which only 7 were selected as potential markers of VIT efficiency because they maintained their significant differences throughout the normalization process. For these reasons we consider that the signification detected for these citokines is robust enough. However, we also would like to mention that this is an exploratory study that aims to identify potential markers and encourage future studies in this direction that can enrich the knowledge in this field and confirm our findings.

In conclusion, this information may be useful to establish a correlation between the variation in the level of expression of the cytokines and the efficiency of the immunotherapy treatment, with the resulting applications in the field of research and diagnosis. In addition, this optimization gives important information regarding to characterization of patients and response to immunotherapy.

It has been claimed that there is an urgent need to identify and validate predictive biomarkers that can be used to monitor allergen immunotherapy and novel assays/biomarkers are currently under development [14]. To the best of our knowledge this is the first cytokine array on successful immunotherapy with hymenoptera venom. This approach could allow a better knowledge of the mechanisms of immunotherapy and could also contribute to the development of predictive biomarkers of successful immunotherapy.

\section{ACKNOWLEDGEMENTS}

This work was supported by a grant of the "Fundación para la Investigación de la Sociedad Española de Alergología e Inmunología Clínica".

\section{CONFLICT OF INTEREST}

The authors of this work declare that they have no conflict of interest

\section{ABBREVIATIONS}

$$
\begin{array}{ll}
\mathrm{CV} & =\text { Coefficients of variation } \\
\mathrm{DC} & =\text { Dendritic cell }
\end{array}
$$




\section{REFERENCES}

[1] Devey ME, Lee SR, Richards D, Kemeny DM. Serial studies on the functional affinity and heterogeneity of antibodies of different IgG subclasses to phospholipase A2 produced in response to beevenom immunotherapy. J Allergy Clin Immunol 1989; 84: 326-30.

[2] Jutel M, Pichler WJ, Skrbic D, Urwyler A, Dahinden C, Muller UR. Bee venom immunotherapy results in decrease of IL-4 and IL5 and increase of IFN-gamma secretion in specific allergenstimulated T cell cultures. J Immunol 1995;154: 4187-94.

[3] Segura JA, Assenmacher M, Irsch J, Hunzelmann N, Radbruch A. Systemic T-cell unresponsiveness during rush bee-venom immunotherapy. Allergy 1998; 53: 233-40.

[4] Bellinghausen I, Metz G, Enk AH, Christmann S, Knop J, Saloga J. Insect venom immunotherapy induces interleukin-10 production and a Th2-to-Th1 shift, and changes surface marker expression in venom-allergic subjects. Eur J Immunol 1997; 27: 1131-9.

[5] Mamessier E, Birnbaum J, Dupuy P, Vervloet D, Magnan A. Ultrarush venom immunotherapy induces differential $\mathrm{T}$ cell activation and regulatory patterns according to the severity of allergy. Clin Exp Allergy 2006; 36: 704-13.

[6] Schena M, Shalon D, Davis RW, Brown PO. Quantitative monitoring of gene expression patterns with a complementary DNA microarray. Science 1995; 270: 467-70.

[7] Lockhart DJ, Dong H, Byrne MC, et al. Expression monitoring by hybridization to high-density oligonucleotide arrays. Nat Biotechnol 1996; 14: 1675-80.

[8] Marshall A, Hodgson J. DNA chips: an array of possibilities. Nat Biotechnol 1998; 16: 27-31.

[9] Haab BB, Dunham MJ, Brown PO. Protein microarrays for highly parallel detection and quantitation of specific proteins and antibodies in complex solutions. Genome Biol 2001; 2: 4-13.

[10] Harwanegg C, Laffer S, Hiller R, et al. Microarrayed recombinant allergens for diagnosis of allergy. Clin Exp Allergy 2003; 33: 7-13.

[11] Hiller R, Laffer S, Harwanegg C, et al. Microarrayed allergen molecules: diagnostic gatekeepers for allergy treatment. FASEB J 2002; 16: 414-6.

[12] Kim TE, Park SW, Cho NY, et al. Quantitative measurement of serum allergen-specific IgE on protein chip. Exp Mol Med 2002; 34: 152-58.

[13] Jahn-Schmid B, Harwanegg C, Hiller R, et al. Allergen microarray: comparison of microarray using recombinant allergens with conventional diagnostic methods to detect allergen-specific serum immunoglobulin E. Clin Exp Allergy 2003; 33: 1443-9.

[14] James LK, Durham SR. Update on mechanisms of allergen injection immunotherapy. Clin Exp Allergy 2008; 38: 1074-88.

[15] Bilo BM, Rueff F, Mosbech H, Bonifazi F, Oude-Elberink JN. Diagnosis of Hymenoptera venom allergy. Allergy 2005; 60: 133949.

[16] Bonifazi F, Jutel M, Bilo BM, Birnbaum J, Muller U. Prevention and treatment of hymenoptera venom allergy: guidelines for clinical practice. Allergy 2005; 60: 1459-70.

[17] Isidoro-García M, Dávila I, Laffond E, Moreno E, Lorente F, González-Sarmiento R. Interleukin-4 (IL4) and Interleukin-4 receptor (IL4RA) polymorphisms in asthma: a case control study. Clin Mol Allergy 2005; 3: 15.

[18] Hamelinck D, Zhou H, Li L, et al. Optimized normalization for antibody microarrays and application to serum-protein profiling. Mol Cell Proteomics 2005; 4: 773-84

[19] De Amici M, Puggioni F, Casali L, Alesina R. Variations in serum levels of interleukin (IL)-1beta, IL-2, IL-6, and tumor necrosis factor-alpha during specific immunotherapy. Ann Allergy Asthma Immunol 2001; 86: 311-3.

[20] Jerzynska J, Stelmach W, Majak P, Brzozowska A, Sobocinska A, Stelmach I. Effect of specific immunotherapy on serum levels of tumor necrosis factor alpha in asthmatic children. Allergy Asthma Proc 2008; 29: 274-9.

[21] Knight J. When the chips are down. Nature 2001; 410: 860-1.

[22] Marshall E. Getting the noise out of gene arrays. Science 2004; 306: 630-1.

[23] Levings M.K, Sangregorio R, Galbiati F, Squadrone S, Malefyt $\mathrm{RD}$, Roncarolo MG. IFN-alpha and IL-10 induce the differentiation of human type $1 \mathrm{~T}$ regulatory cells. J Immunol 2001; 166: 5530-39.

[24] Akdis M, Akdis CA. Mechanisms of allergen-specific immunotherapy. J Allergy Clin Immunol 2007; 119: 780-89.

[25] Akdis CA, Blesken T, Akdis M, Wuthrich B, Blaser K. Role of interleukin 10 in specific immunotherapy. J Clin Invest 1998; 102: 98-106.

[26] Karjalainen J, Virta M, Pessi T, Hulkkonen J, Nieminen MM, Hurme M. Childhood cat exposure-related tolerance is associated with IL1A and IL10 polymorphisms. J Allergy Clin Immunol 2005; 116: 223-5.

[27] Campbell IK, Ianches G, Hamilton JA. Production of macrophage colony-stimulating factor (M-CSF) by human articular cartilage and chondrocytes. Modulation by interleukin-1 and tumor necrosis factor alpha. Biochim Biophys Acta 1993; 1182: 57-63.

[28] Libby P, Sukhova G, Lee RT, Galis ZS. Cytokines regulate vascular functions related to stability of the atherosclerotic plaque. J Cardiovasc Pharmacol 1995; 25(2): 9-12.

[29] Kwan WH, Boix C, Gougelet N, Fridman WH, Mueller CG. LPS induces rapid IL-10 release by $\mathrm{M}$-CSF-conditioned tolerogenic dendritic cell precursors. J Leukoc Biol 2007; 82: 133-41.

[30] Lo AS, Taylor JR, Farzaneh F, Kemeny DM, Dibb NJ, Maher J. Harnessing the tumour-derived cytokine, CSF-1, to co-stimulate Tcell growth and activation. Mol Immunol 2008; 45: 1276-87.

[31] Boulay ME, Boulet LP. Airway response to low-dose allergen exposure in allergic nonasthmatic and asthmatic subjects: eosinophils, fibronectin, and vascular endothelial growth factor. Chest 2003; 123: 430S.

[32] Ciprandi G, Colombo BM, Murdaca G, De Amici M. Serum vascular endothelial growth factor and sublingual immunotherapy. Allergy 2008; 63: 945-6.

[33] Honda K, Arima M, Cheng G, et al. Prostaglandin D2 reinforces Th2 type inflammatory responses of airways to low-dose antigen through bronchial expression of macrophage-derived chemokine. J Exp Med 2003; 198: 533-43.

[34] Imai T, Nagira M, Takagi S, et al. Selective recruitment of CCR4bearing Th2 cells toward antigen-presenting cells by the $\mathrm{CC}$ chemokines thymus and activation-regulated chemokine and macrophage-derived chemokine. Int Immunol 1999; 11: 81-8.

[35] Struyf S, Proost P, Sozzani S, et al. Cutting edge: enhanched antiHIV-1 activity and altered chemotactic potency of NH2-terminally processed macrophage-derived chemokine (MDC) imply an additional MDC receptor. J Immunol 1998; 161: 2672-5.

[36] Proost P, Struyf S, Schols D, et al. Truncation of macrophagederived chemokine by CD26/ dipeptidyl-peptidase IV beyond its predicted cleavage site affects chemotactic activity and CC chemokine receptor 4 interaction. J Biol Chem 1999; 274: 3988-93.

[37] Mantovani A, Gray PA, Van Damme J, Sozzani S. Macrophagederived chemokine (MDC). J Leukoc Biol 2000; 68: 400-4.

[38] Huang E, Nocka K, Beier DR, et al. The hematopoietic growth factor $\mathrm{KL}$ is encoded by the Sl locus and is the ligand of the c-kit receptor, the gene product of the W locus. Cell 1990; 63: 225-33.

[39] Yee NS, Langen H, Besmer P. Mechanism of kit ligand, phorbol ester, and calcium-induced down-regulation of c-kit receptors in mast cells. J Biol Chem 1993; 268: 14189-201.

[40] Krishnamoorthy N, Oriss TB, Paglia M, et al. Activation of c-Kit in dendritic cells regulates $\mathrm{T}$ helper cell differentiation and allergic asthma. Nat Med 2008; 14: 565-73. 